\title{
Higher-Order Contingentism, Part 3: Expressive Limitations*
}

\author{
Peter Fritz
}

Final Draft

\begin{abstract}
Two expressive limitations of an infinitary higher-order modal language interpreted on models for higher-order contingentism - the thesis that it is contingent what propositions, properties and relations there are are established: First, the inexpressibility of certain relations, which leads to the fact that certain model-theoretic existence conditions for relations cannot equivalently be reformulated in terms of being expressible in such a language. Second, the inexpressibility of certain modalized cardinality claims, which shows that in such a language, higher-order contingentists cannot express what is communicated using various instances of talk of 'possible things', such as 'there are uncountably many possible stars'.
\end{abstract}

\section{Introduction}

Part 1 (Fritz and Goodman, 2016) explores several variants of a higher-order contingentist theory, there called the Fine-Stalnaker view. This paper continues this exploration, and assumes that the reader is familiar with Part 1, although familiarity with Part 2 (Fritz, forthcoming) is not needed. This third part is concerned with two questions which arise from expressive limitations of the infinitary higher-order modal language introduced in Part 1. The first is the question how to state the Fine-Stalnaker view, continuing the discussion of this issue started in Part 1. The second is the question what claims about possible individuals can be expressed if the Fine-Stalnaker view is correct; the formal results on this issue to be established here serve as central premises in a philosophical evaluation of higher-order contingentism in Fritz and Goodman (forthcoming). Before moving on to introducing the philosophical background to the technical questions to be explored, recall that even though the models developed in Part 1 are standard possible worlds models, it is not assumed that necessarily equivalent relations are identical, and that therefore, the quantifiers of the language to be used are read as implicitly restricted to hereditarily intensional relations, as discussed in Part 1, section 3.4. This qualification will mostly be left tacit in the following.

*Forthcoming in the Journal of Philosophical Logic. The final publication is available at https://link. springer. com/article/10.1007/s10992-017-9443-0. 


\subsection{Stating the Views}

The investigation of theories of higher-order contingency in Part 1 starts with two variants of the Fine-Stalnaker view, called the higher-order closure view and the qualitative generation view. Formally, the class of closed models $C \times$ is developed, where $x$ is a parameter which indicates whether a positive or negative semantics is used. It is argued that this class of models does not capture the views expressed in the philosophical writings that serve as its motivation. In the case of closure, this is spelled out formally by showing that the comprehension principle $\times \operatorname{Comp}_{F S}$ is not valid on $C \times$. In response to this problem, a more restrictive class of models is introduced which validates $\times \operatorname{Comp}_{F S}$, but this class of models turns out to be so restrictive as to rule out models that are paradigmatic instances of the informal picture-thinking that motivated the views.

In order to keep the original model theory, one might reject the way the basic idea underlying the Fine-Stalnaker view was spelled out in terms of automorphisms. Instead, one might propose to spell it out by formulating the existence condition for relations linguistically. The higher-order closure view would then be cashed out as saying that necessarily, a relation exists if it is expressible in principle using only existing parameters, and the qualitative generation view as saying that necessarily, a relation exists if and only if it is expressible in principle using only generating parameters. (Here, an existing/generating parameter is an expression which is interpreted as a relation which, in the world in question, exists/is among the choice of relations from which the higher-order domains are generated.)

While the notion of expressibility in principle - given certain parameters - is somewhat unclear, it is not uncommon to find philosophers appeal to it. E.g., as observed in Stalnaker (2012, p. 61), it is used in explications of the notion of qualitativeness of relations in Adams (1979, p. 7) and Lewis (1986, p. 221). Concerning the present issue of formulating a theory of higher-order contingentism, the idea is discussed in Fine (1977b, section V). Adams, Lewis and Fine all seem to suggest that expressibility in principle can be understood as expressibility in a sufficiently rich language, but only Fine is more specific about what such a language might look like. Fine in fact specifies an infinitary language very similar to the one used in Part 1 , and proves that his semantic criterion of the existence of relations in terms of automorphisms coincides with the linguistic criterion of being expressible in his infinitary language using only generating parameters. Fine's result is therefore a promising sign for formulating the higher-order closure and qualitative generation views linguistically.

However, as will be shown here, Fine's result essentially depends on a questionable resource to which he avails himself, namely a primitive "outer" firstorder quantifier binding infinite sets of variables, which he writes $\exists \triangleright$. Understood primitively, such a quantifier is highly suspect from the point of view of a contingentist. Fine's explanation of it on p. 161 suggests that it can be understood as an infinite sequence of possibility operators and existential quantifiers, and this understanding is supported further by the fact that in other writings on the subject, Fine explicitly appeals to such embeddings; see Fine (1977a) and Fine (2003). Infinitary embeddings of this kind are not allowed in the infinitary language used in Part 1. The first main result to be established here is that without these resources of infinitary outer quantification or infinitary embeddings, the 
analogs to Fine's result fail: In the case of closure, it will be shown that there are models in which necessarily, every relation expressible in the language of Part 1 using existing parameters exists but which are not closed. This is established in section 2 . It would be relatively straightforward to adapt this result to the case of qualitative generation, using the extensions of syntax and semantics sketched in Part 1, section 6.5. For simplicity, the following will consider only the case of closure. Languages with infinitary embeddings will be discussed in section 4.1.

The condition on models that necessarily, every relation expressible using existing parameters exists is equivalent to validating the comprehension principle $\times \operatorname{Comp}_{C}$, as will be proven in Proposition 2.2. Since $\times \operatorname{Comp}_{C}$ is valid on $C \times$, the result to be proven can be restated as saying that $\times \operatorname{Comp}_{C}$ does not define the class $C \times$, in the sense that it is not the case for every model that it validates $\times$ Comp $_{C}$ if and only if it is in $C \times$; this results also holds when only world-selective models are considered. In fact, the way the result is proven establishes something stronger, namely that no class of sentences defines $C \times$ (Corollary 2.15).

These results show that in formulating the higher-order closure and qualitative generation views of higher-order contingency, one cannot simply assume that being expressible in principle can be cashed out as being expressible in a particular language which provides the required infinitary resources; what is expressible depends on subtle issues concerning which infinitary resources are available. Of course, this does not mean that $\times \operatorname{Comp}_{F S}$ (or its analog for generation), which commits one to the more restrictive model theory of internally closed or internally generated models, is the only way of formulating a theory of higher-order contingency in the vicinity of the Fine-Stalnaker view. E.g., one might hold the view that necessarily, a relation exists just in case all possible individuals it is about exist. ${ }^{1}$ However, such a view is a significant departure from the guiding ideas behind the Fine-Stalnaker view, and in particular from the reductive ambitions which seem to lie at the heart of Fine (1977b).

\subsection{Paraphrase}

In Fritz and Goodman (forthcoming), it is argued that claims which seem to be quantifying over merely possible individuals, such as the claim that there are possible buildings which have never and will never be built, convey a proposition other than the one they (literally) express. Such claims are trivially false according to the contingentist's metaphysics but seem to convey something true, so it is argued that contingentists must provide paraphrases which (literally) express the proposition these claims convey. In the case just mentioned, this is easily done by saying that there could have been buildings which actually have never and will never be built. It is shown there that using even highly infinitary first-order resources, analogous modalized cardinality claims such as the claim that most possible people are never born cannot be paraphrased. Whether such claims can be paraphrased using infinitary higher-order resources is the second issue of expressivity of this part, which is the topic of section 3 .

A positive expressivity result will be sketched and a negative expressivity result will be proven. The positive result is that assuming first-order contingen-

\footnotetext{
${ }^{1}$ This was suggested by Kit Fine (p.c.). As he noted, this proposal also brings the existence condition for relations more in line with that of the extensional entities treated in Fine (1977b), but omitted in the present type hierarchy.
} 
tism but higher-order necessitism, any claim formulated with modalizations of generalized quantifiers can be paraphrased using the corresponding unmodalized quantifiers on properties restricted to certain haecceities. The negative result is that using either the class of closed or internally closed models (whether positive or negative), the claim that there are at least $\kappa$ many possible individuals, for a given uncountable cardinality $\kappa$, is inexpressible. Again, the results can easily be adapted to the cases of generation and internal generation, but the following focuses on closure for simplicity. On the basis of these results, Fritz and Goodman (forthcoming) argue against the versions of higher-order contingentism discussed here.

Before proceeding to proving these limitative results in sections 2 and 3, the main tool for doing so will be defined, namely back and forth systems. In section 4 , some possible extensions of the formal object language are considered and it is discussed how likely they are to overcome the expressive limitations discussed here.

\subsection{Back and Forth Systems}

The central tool in proving the limitative results of this paper are back and forth systems. Although their definition is somewhat complex, they are straightforward extensions of well-known definitions; see Fritz (2013, section 2.2) for references. It may be of historical interest that back and forth systems for higherorder logics were in fact defined relatively early; they go back at least to Fraïssé (1958). As in Fritz (2013), the back and forth systems used here connect worlds of two models, requiring connected worlds to be isomorphic. Whereas in Fritz (2013), this was done merely for simplicity, it is essential here, since roughly, any world can be described up to isomorphism using a sentence of infinitary higher-order modal logic. Similar to the familiar notion of a bisimulation - see Blackburn et al. (2001, section 2.2), the only closure condition on back and forth systems here corresponds to the behaviour of modal operators, effectively interpreted using an implicit universal accessibility relation on worlds.

Recall that for a function $f$ from a set $A$ to a set $B, \operatorname{dom}(f)$ is the domain of $f . \operatorname{im}(f)$ is now written for the image of $f$, the set $\{y \in B: f(x)=y$ for some $x \in A$ \}. When convenient, functions are considered as functional relations. For the rest of the paper, a choice of a signature $\sigma$ and sign $\times$ will tacitly be assumed.

Definition 1.1. Let $\mathfrak{M}=\langle W, I, D, V, w\rangle$ and $\mathfrak{M}^{\prime}=\left\langle W^{\prime}, I^{\prime}, D^{\prime}, V^{\prime}, w^{\prime}\right\rangle$ be models. A partial isomorphism from $\mathfrak{M}$ to $\mathfrak{M}^{\prime}$ is a tuple $\langle\tau, \rho\rangle$ such that

- $\tau$ is a partial injection from $W$ to $W^{\prime}$

- $\rho$ is a function on types mapping each type $t$ to a bijection $\rho^{t}$ from $D_{\operatorname{dom}(\tau)}^{t}$ to $D_{\operatorname{im}(\tau)}^{\prime t}$ such that for all $v \in \operatorname{dom}(\tau)$ :

- for all types $t, \rho^{t} \mid D_{v}^{t}$ is a bijection from $D_{v}^{t}$ to $D_{\tau(v)}^{\prime t}$

- for all types $\bar{t}$, for all $o \in D_{\operatorname{dom}(\tau)}^{t}$ and $\bar{o} \in \Pi_{i \leq n} D_{\operatorname{dom}(\tau)}^{t_{i}}, \bar{o} \in o(v)$ iff $\left\langle\rho^{t_{i}}\left(o_{i}\right): i \leq n\right\rangle \in \rho^{t}(o)(\tau(v))$

- $\tau(w)=w^{\prime}$ 
- for all types $t$ and $a \in \sigma(t), \rho^{t} V(a)=V^{\prime}(a)$

Let a back and forth system from $\mathfrak{M}$ to $\mathfrak{M}^{\prime}$ be a non-empty set $J$ of partial isomorphisms from $\mathfrak{M}$ to $\mathfrak{M}^{\prime}$ such that for all $\langle\tau, \rho\rangle \in J$ :

- For all $v \in W$, there is a $\left\langle\tau^{\prime}, \rho^{\prime}\right\rangle \in J$ such that $\tau \subseteq \tau^{\prime}$ and $\rho^{t} \subseteq \rho^{\prime t}$ for all types t, and $v \in \operatorname{dom}\left(\tau^{\prime}\right)$.

- For all $v^{\prime} \in W^{\prime}$, there is a $\left\langle\tau^{\prime}, \rho^{\prime}\right\rangle \in J$ such that $\tau \subseteq \tau^{\prime}$ and $\rho^{t} \subseteq \rho^{\prime t}$ for all types $t$, and $v^{\prime} \in \operatorname{im}\left(\tau^{\prime}\right)$.

Write $J: \mathfrak{M} \cong \infty \mathfrak{M}^{\prime}$ for $J$ being a back and forth system from $\mathfrak{M}$ to $\mathfrak{M}^{\prime}$ and $\mathfrak{M} \cong \infty \mathfrak{M}^{\prime}$ for there being a $J$ such that $J: \mathfrak{M} \cong \infty \mathfrak{M}^{\prime}$.

Proposition 1.2. For any models $\mathfrak{M}$ and $\mathfrak{M}^{\prime}$ of the same signature,

if $\mathfrak{M} \cong \infty \mathfrak{M}^{\prime}$ then $\mathfrak{M} \equiv \mathfrak{M}^{\prime}$.

Proof. By induction on the complexity of formulas.

\section{Expressing Relations}

The first task is to make the claim to be proven precise. Focussing on the case of closure, it will be shown that there are models in which the semantic criterion of closure comes apart from the syntactic criterion of necessarily containing every relation expressible using existing parameters. The next definition therefore formalizes the latter criterion, calling it expressible closure, using the notion of a formula expressing a relation introduced in Part 1, Definition 20. (For alternative conceptions of expressibility, see Fine (1977b, pp. 162-163). The notion used here is both natural from a conceptual point of view and useful from a technical point of view.)

Definition 2.1. Let $\mathfrak{M}=\langle W, I, D, V, w\rangle$ be a model and $\bar{t}$ a sequence of types.

Define any $o \in \iota_{\langle W, I\rangle}^{\bar{t}}$ to be xexpressible in $v \in W$ if there is a formula $\varphi$ of $\mathcal{L}(\emptyset)$, sequence of variables $\bar{x}$ of types $\bar{t}$ and assignment a for $\langle W, I\rangle$ admissible for $\varphi$ such that $\operatorname{im}(a) \subseteq D_{v}^{T}$ and $o=\varphi(\bar{x})_{\mathfrak{M}, a}^{\times}$.

$\mathfrak{M}$ is expressibly $\times$ closed if for all $v \in W, D_{v}^{T}$ contains all $o \in \iota_{\langle W, I\rangle}^{T}$ which are $\times$ expressible in $v$.

As noted above, being expressibly closed is equivalent to verifying Comp : $_{C}$ :

Proposition 2.2. A model $\mathfrak{M}$ is expressibly $\times$ closed if and only if $\mathfrak{M} \vDash \times \mathrm{Comp}_{C}$.

Proof. Immediate.

Since by Part 1 , Proposition 8 , every $\times$ closed model verifies $\times \mathrm{Comp}_{C}$, it follows that every $\times$ closed model is expressively $\times$ closed. It will therefore have to be shown that some expressively $\times$ closed model is not $\times$ closed. To do so, a model will be considered in which there is a set of worlds which share a certain individual, and a set of worlds which share a different individual. One of the two sets of worlds is countably infinite and the other uncountably infinite. Any automorphism must map any two worlds sharing an individual to worlds sharing a corresponding individual, so for cardinality reasons, any automorphism must 
map each world of the uncountable set to a world of the same set. Similarly, any automorphism must map any two worlds with distinct individuals to worlds with corresponding distinct individuals, so no world of the countable set may be mapped to a world of the uncountable set. Consequently, every automorphism maps each of the countable and uncountable sets to itself. Closure therefore forces the two propositions corresponding to these two sets of worlds to exist at the distinguished world. However, by letting the individual domain of this world be empty, the model can be constructed in such a way that these propositions are not expressible at that world, which yields an expressibly closed model which is not closed.

The difficult part of this proof is specifying the higher-order domains of the model in a way which guarantees that the model is expressibly closed. To do so, a similar model is first defined in which the two sets of worlds - distinguished by the individuals the respective worlds share - are both countably infinite; to ensure that this model is closed, it is constructed by generation. The higherorder domains of this model are then projected onto the frame of the model to be constructed using a technique which will now be developed, and called projective generation. (While this is a highly specific technique, it will also be useful in section 3.) Projective generation leads to a back and forth system from the original model to the one which is projectively generated. Since the original model is generated, it is closed, and therefore verifies $\times \operatorname{Comp}_{C}$. So by the equivalence of models related by a back and forth system, the projectively generated model also verifies $\times \operatorname{Comp}_{C}$, and so by Proposition 2.2 is expressibly closed. That it is not closed can easily be established by showing that the two propositions indicated earlier are not in the higher-order domain of the distinguished world.

\subsection{Projective Generation}

Projective generation deals only with structures which are determined by their worlds and the distribution of individuals at the worlds, and in which all worlds only contain finitely many individuals:

Definition 2.3. Let a finitary individual structure, in short FIS, be a structure $\mathfrak{S}=\langle W, I, D\rangle$ such that

(i) for all types $t \neq e, D_{W}^{t}=\emptyset$,

(ii) for all $w \in W, w=D_{w}^{e}$, and

(iii) for all $w \in W, D_{w}^{e}$ is finite.

In the context of FISs, both automorphisms of a structure and partial isomorphisms, as used in back and forth systems between models, can be reduced to partial injections between individuals. The relevant partial injections are singled out in the following definition:

Definition 2.4. Let $\mathfrak{S}=\langle W, I, D\rangle$ and $\mathfrak{S}^{\prime}=\left\langle W^{\prime}, I^{\prime}, D^{\prime}\right\rangle$ be FISs and $f$ a partial injection from $I$ to $I^{\prime}$.

- $f$ respects worlds if for all $X \subseteq \operatorname{dom}(f), X \in W$ iff $\{f(x): x \in X\} \in W^{\prime}$. 
- If $f$ respects worlds, let $\dot{f}$ be the partial function from $W$ to $W^{\prime}$ mapping each $w \in W$ such that $w \subseteq \operatorname{dom}(f)$ to $\{f(x): x \in w\}$, and $\hat{f}=\langle\dot{f}, f\rangle$. Note that $\dot{f}$ is injective.

This definition is slightly sloppy as the relativity to the structures is not noted, but context will make this clear in all applications below. It will now be shown how automorphisms of a structure - consisting of a permutation of worlds and a permutation of individuals - can be reduced to a permutation of individuals. To state this, recall that if a group $G$ acts on some set $X$ and $x \in X$, $G_{x}$ is the stabilizer subgroup of $x$, the set of $g \in G$ which map $x$ to itself. This notion is now extended to sets: for any $Y \subseteq X$, write $G_{(Y)}$ for the point-wise stabilizer subgroup of $Y$, the set of $g \in G$ which map each $x \in Y$ to itself.

Definition 2.5. Let $\mathfrak{S}=\langle W, I, D\rangle$ be an FIS and $w \in W$. Define:

- $\operatorname{aut}^{i}(\mathfrak{S})=\left\{f \in S_{I}: f\right.$ respects worlds $\}$

- $\operatorname{fix}^{i}(\mathfrak{S}, w)=\operatorname{aut}^{i}(\mathfrak{S})_{(w)}$

Lemma 2.6. For any FIS $\mathfrak{S}=\langle W, I, D\rangle$ and $w \in W$, aut(S) $=\{\hat{f}: f \in$ $\left.\operatorname{aut}^{i}(\mathfrak{S})\right\}$ and $\operatorname{fix}(\mathfrak{S}, w)=\left\{\hat{f}: f \in \operatorname{fix}^{i}(\mathfrak{S}, w)\right\}$.

Proof. Immediate.

For the definition of projective generation, a class of special FISs is further singled out, inspired by the notion of homogeneity in model theory, as defined, e.g., in Hodges (1997, p. 160). To define it, let a partial permutation of a set $X$ be a partial injection from $X$ to $X$. It should be noted that the present notion of homogeneity is unrelated to the notion of homogeneity discussed in Fine (1977b, p. 150); a notion similar to Fine's notion of homogeneity is discussed in section 3.2 under the label of being "fully symmetric".

Definition 2.7. An FIS $\mathfrak{S}=\langle W, I, D\rangle$ is homogeneous if each finite partial permutation of $I$ which respects worlds can be extended to a permutation of I which respects worlds, i.e., an element of $\operatorname{aut}^{i}(\mathfrak{S})$.

Projective generation makes it possible to project the higher-order domains of a structure generated from a homogeneous FIS onto an FIS. This will be done relative to a construction connecting the first to the second FIS, which will be called a projection. Projections can be thought of as back and forth systems from the first to the second FIS which satisfy certain additional conditions. In the following definition, conditions (i) and (ii) correspond to the conditions on back and forth systems; condition (iii) encodes the idea that the projection maps every world of the first FIS to a world of the second FIS; and condition (iv) encodes the idea that a projection must cohere with the automorphisms of the first structure. For condition (iv), partial functions and total functions are composed just as relations in general; i.e., $f g=\{\langle x, z\rangle$ : there is a $y$ such that $\langle x, y\rangle \in g$ and $\langle y, z\rangle \in f\}$.

Definition 2.8. Let $\mathfrak{S}=\langle W, I, B\rangle$ be an homogeneous FIS and $\mathfrak{S}^{\prime}=\left\langle W^{\prime}, I^{\prime}, B^{\prime}\right\rangle$ an FIS. A projection from $\mathfrak{S}$ to $\mathfrak{S}^{\prime}$ is a set $P$ of finite partial injections from $I$ to $I^{\prime}$ which respect worlds such that for all $p \in P$ :

(i) For all $w \in W$, there is a $q \in P$ such that $p \subseteq q$ and $w \subseteq \operatorname{dom}(q)$. 
(ii) For all $w^{\prime} \in W^{\prime}$, there is a $q \in P$ such that $p \subseteq q$ and $w^{\prime} \subseteq \operatorname{im}(q)$.

(iii) For all $w \in \operatorname{dom}(\dot{p}), p \mid w \in P$.

(iv) For all $f \in \operatorname{aut}^{i}(\mathfrak{S}), p f \in P$.

Members of $P$ are called perspectives.

For the rest of this section, assume that $\mathfrak{S}, \mathfrak{S}^{\prime}$ and $P$ are given as in Definition 2.8. Let $\otimes \mathfrak{S}=\langle W, I, D\rangle, \mathfrak{F}=\langle W, I\rangle$ and $\mathfrak{F}^{\prime}=\left\langle W^{\prime}, I^{\prime}\right\rangle$. Using $P$, the domain assignment of $\otimes \mathfrak{S}$ will be projected onto $\mathfrak{F}^{\prime}$; this will coincide with $\mathfrak{S}^{\prime}$ for individuals, so one can also think of $P$ as projecting the higher-order domains of $\otimes \mathfrak{S}$ onto $\mathfrak{S}^{\prime}$.

The definition to be given simultaneously defines, first, a relation $Z$, parametric to types and perspectives, from $D$ to intensions on $\mathfrak{F}^{\prime}$ and, second, a domain assignment $D^{P}$ on $F^{\prime}$. The idea behind this definition is to extend any perspective $p$ to a connection between intensions corresponding to relations among individuals: An intension $o$ in the domain of $\otimes \mathfrak{S}$ is connected to an intension $o^{\prime}$ on $\mathfrak{F}^{\prime}$ just in case for every perspective $q$ extending $p, o$ and $o^{\prime}$ agree on individuals related by $q$ in worlds related by $q$. This extension of $P$ can then be used to define the domains of intensions corresponding to relations among individuals on $\mathfrak{F}^{\prime}$, including in the domain of a given world $v$ all intensions in the image of each of the extensions of a perspective restricted to the domain of the world which it maps to $v$. Iterating this procedure along the type hierarchy yields the definitions of $Z$ and $D^{P}$. Its statement uses the the convention of writing, for a binary relation $R \subseteq X \times Y$ and $Z \subseteq X, R[Z]$ for the image of $Z$ under $R$, i.e., the set $\{y:\langle x, y\rangle \in R$ for some $x \in Z\}$.

Definition 2.9. Define a relation $Z_{p}^{t} \subseteq D_{\mathrm{dom}(\dot{p})}^{t} \times \iota_{\widetilde{\mathfrak{F}}^{\prime}}^{t}$ for each type $t$ and $p \in P$ and a domain assignment $D^{P}$ for $\mathfrak{F}^{\prime}$ by simultaneous induction on types:

$t=e:$ For all $p \in P, Z_{p}^{e}=p \mid D_{\operatorname{dom}(\dot{p})}^{e}$.

For all $v \in W^{\prime},\left(D^{P}\right)_{v}^{e}=B_{v}^{\prime e}$.

$t=\bar{t}:$ For all $p \in P, o \in D_{\operatorname{dom}(\dot{p})}^{t}$ and $o^{\prime} \in \iota_{\mathfrak{F}^{\prime}}^{t}, o Z_{p}^{t} o^{\prime}$ iff

(1) $D^{P} \otimes o^{\prime}$ and

(2) for all $q \in P$ such that $p \subseteq q, w \in \operatorname{dom}(\dot{q})$ and $n$-tuples $\bar{o}, \bar{o}^{\prime}$ such that $o_{i} Z_{q}^{t_{i}} o_{i}^{\prime}$ for all $i \leq n, \bar{o} \in o(w)$ iff $\bar{o}^{\prime} \in o^{\prime}(\dot{q}(w))$.

For all $v \in W^{\prime}, D_{v}^{P^{t}}=\bigcup\left\{Z_{p}^{t}\left[D_{\dot{p}^{-1}(v)}^{t}\right]: p \in P\right.$ and $\left.v \in \operatorname{im}(\dot{p})\right\}$.

For brevity, $\bar{o} Z_{p} \bar{o}^{\prime}$ will be written for the claim that $o_{i} Z_{p}^{t_{i}} o_{i}^{\prime}$ for all $i \leq n$. Let the structure projectively $\times$ generated by $P$ be $\mathfrak{S}^{P}=\left\langle W^{\prime}, I^{\prime}, D^{P}\right\rangle$.

This is well-defined since $D^{P}$ only needs to be defined for lower types to evaluate whether $D^{P} \otimes o^{\prime}$.

It now needs to be shown that $Z$ is well-behaved. The main claim to be established is that all relations $Z_{p}^{t}$ are bijections, which is proven in the next lemma. It turns out to be convenient to prove also, by simultaneous induction, that if two perspectives agree on the individuals of the generating structure, their extensions agree on the higher-order domains of that world as well. 
Lemma 2.10. For all types $t$ and $p \in P$ :

(i) $Z_{p}^{t}$ is a bijection from $D_{\operatorname{dom}(\dot{p})}^{t}$ to $\left(D^{P}\right)_{\operatorname{im}(\dot{p})}^{t}$.

(ii) For all $q \in P, w \in W$ such that $w \subseteq \operatorname{dom}(p \cap q)$ and $o \in D_{w}^{t}, Z_{p}^{t}(o)=$ $Z_{q}^{t}(o)$

Since the proof of this lemma is somewhat involved, it is given in Appendix A. An immediate consequence of this which will be useful several times below is the following lemma, which says that extending a perspective to relations and then restricting this to the domain of some world yields the same result as first restricting the perspective to that world and then extending this to relations:

Lemma 2.11. For any type $t, p \in P$ and $w \in \operatorname{dom}(\dot{p}), Z_{p}^{t} \mid D_{w}^{t}=Z_{p \mid w}^{t}$.

Proof. By condition (iii) of the definition of projections, $p \mid w \in P$. If $\left\langle o, o^{\prime}\right\rangle \in$ $Z_{p \mid w}^{t}$, then by Lemma 2.10 (i), there is a $v \in \operatorname{dom}(\dot{p})$ such that $v \in W$ and $o \in D_{v}^{t}$. Since $v \subseteq w$, $\operatorname{fix}(\mathfrak{S}, w) \subseteq \operatorname{fix}(\mathfrak{S}, v)$, so $D_{v}^{t} \subseteq D_{w}^{t}$, and thus $o \in D_{w}^{t}$. By Lemma 2.10 (ii), $Z_{p}^{t}(o)=Z_{p \mid w}^{t}(o)$, so $\left\langle o, o^{\prime}\right\rangle \in Z_{p}^{t} \mid D_{w}^{t}$. If $\left\langle o, o^{\prime}\right\rangle \in Z_{p}^{t} \mid D_{w}^{t}$, then $o \in D_{w}^{t}$, so by Lemma $2.10(\mathrm{i}), o \in \operatorname{dom}\left(Z_{p \mid w}^{t}\right)$, and so by Lemma 2.10 (ii), $Z_{p}^{t}(o)=Z_{p \mid w}^{t}(o)$, and therefore $\left\langle o, o^{\prime}\right\rangle \in Z_{p \mid w}^{t}$.

Finally, the construction is extended from structures to models. Consider a model $\mathfrak{M}=\langle W, I, D, V, w\rangle$ on $\otimes \mathfrak{S}$. The following definition extends the generation of $\mathfrak{S}^{P}$ to a model, relative to a perspective, and defines the corresponding back and forth system between the two models:

Definition 2.12. For any $p \in P$ such that $w \subseteq \operatorname{dom}(p)$, let the model projectively $\times$ generated from $\mathfrak{M}$ by $P$ and $p$ be $\mathfrak{M}_{p}^{P}=\left\langle W^{\prime}, I^{\prime}, D^{P}, V_{p}^{P}, \dot{p}(w)\right\rangle$, where for all $t \in T$ and $a \in \sigma(t), V_{p}^{P}(a)=Z_{p}^{t} V(a)$.

For every $q \in P$, let $Z_{q}$ be the function on types mapping each type $t$ to $Z_{q}^{t}$. Let $J_{p}^{P}=\left\{\left\langle\dot{q}, Z_{q}\right\rangle: q \in P\right.$ such that $\left.p \subseteq q\right\}$.

By construction, $\mathfrak{M}_{p}^{P}$ is a $\times$ model.

Theorem 2.13. $J_{p}^{P}: \mathfrak{M} \cong \infty \mathfrak{M}_{p}^{P}$.

Proof. Since $P$ is non-empty, so is $J_{p}^{P}$. Consider any $q \in P$; it will first be shown that $\left\langle\dot{q}, Z_{q}\right\rangle$ is a partial isomorphism from $\mathfrak{M}$ to $\mathfrak{M}_{p}^{P}$. Since $q$ respects worlds, $\dot{q}$ is a partial injection from $W$ to $W^{\prime}$. Let $t$ be a type. By Lemma 2.10, $Z_{q}^{t}$ is a bijection from $D_{\operatorname{dom}(\dot{q})}^{t}$ to $D_{\mathrm{im}(\dot{q})}^{\prime t}$. Consider any $v \in \operatorname{dom}(\dot{q})$. By Lemmas 2.10 and 2.11, $Z_{q}^{t} \mid D_{v}^{t}$ is a bijection from $D_{v}^{t}$ to $D_{\dot{q}(v)}^{\prime t}$. So let $t=\bar{t}$ be a type, $o \in D_{\operatorname{dom}(\dot{q})}^{t}$ and $\bar{o} \in \Pi_{i \leq n} D_{\operatorname{dom}(\dot{q})}^{t_{i}}$. Using $Z_{q}^{t}$ both as a relation and function, note that trivially, $\bar{o} Z_{q}\left\langle Z_{q}^{t_{i}}\left(o_{i}\right): i \leq n\right\rangle$, so by construction of $Z, \bar{o} \in o(v)$ iff $\left\langle Z_{q}^{t_{i}}\left(o_{i}\right)\right.$ : $i \leq n\rangle \in Z_{q}^{t}(o)(\dot{q}(v))$, as required. The last two conditions required for $\left\langle\dot{q}, Z_{q}\right\rangle$ being a partial isomorphism from $\mathfrak{M}$ to $\mathfrak{M}_{p}^{P}$ likewise follow straightforwardly from the construction of $\mathfrak{M}_{p}^{P}$ and $J_{p}^{P}$. By conditions (i) and (ii) of the definition of projections and Lemma 2.10 (ii), the required closure conditions on $J_{p}^{P}$ can be established. 


\subsection{Inexpressible Relations}

The next result constructs an expressibly closed model which is not closed. The proof strategy is to construct two FISs, the first containing a countable set of worlds sharing some individual and another countable set sharing another individual, and the second containing a countable set sharing some individual and an uncountable set sharing another individual. Since in FISs, worlds are determined by their individual domains, each world will be given a unique additional individual. More concretely, the following proof will use unordered pairs of ordinals as individual domains: In both FISs, there is a countable set of worlds of the form $\{0, \beta\}$, with $0<\beta<\omega$. In the first FIS, there is another countable set of worlds of the form $\{\omega, \gamma\}$, with $\omega<\gamma<\omega 2(=\omega+\omega)$. In the second FIS, there is an uncountable set of worlds of the form $\{\omega, \gamma\}$, with $\omega<\gamma<\omega_{1}$ (the first uncountable ordinal). Each FIS is completed by the addition of an empty world. From a model based on a structure generated from the first, countable, FIS, a model will be projectively generated on the second, uncountable, FIS. With these constructions, the proof idea outlined at the beginning of this section can be completed. In particular, at the empty world in the countable model, neither proposition corresponding to one of the countable sets sharing one individual (0 or $\omega$ ) will exist, since various automorphisms map one to the other. So as the uncountable model is constructed via the projection just sketched, neither the proposition of worlds containing 0 nor the proposition of worlds containing $\omega$ will exist at the empty world. Thus this model cannot be closed, since for cardinality reasons, no automorphism can map a world of the former proposition to a world of the latter proposition.

Theorem 2.14. There is a world-selective $\times$ model which is expressibly $\times$ closed but not $\times$ closed.

Proof. For any ordinal $\alpha>\omega$, let

$$
W_{2}^{\alpha}=\{\emptyset,\{0, \beta\},\{\omega, \gamma\}: 0<\beta<\omega<\gamma<\alpha\},
$$

and let $\mathfrak{S}_{2}^{\alpha}$ be the unique FIS determined by $W_{2}^{\alpha}$ and $\alpha$. Let $P$ be the set of partial injections $p$ from $\omega 2$ to $\omega_{1}$ with finite domains satisfying the following two conditions:

- For all $\beta \in \operatorname{dom}(p), \beta \in\{0, \omega\}$ iff $p(\beta) \in\{0, \omega\}$.

- For all $\beta, \gamma \in \operatorname{dom}(p),(\beta<\omega$ iff $p(\beta)<\omega)$ iff $(\gamma<\omega$ iff $p(\gamma)<\omega)$.

It is routine to verify that $P$ is a projection from $\mathfrak{S}_{2}^{\omega 2}$ to $\mathfrak{S}_{2}^{\omega_{1}}$. Let $\mathfrak{M}$ be a model based on $\otimes \mathfrak{S}_{2}^{\omega 2}$ with distinguished world $\emptyset$, and $\mathfrak{M}_{\emptyset}^{P}$ the model projectively $\times$ generated from $\mathfrak{M}$ by $P$ and $\emptyset$.

To see that $\mathfrak{M}_{\emptyset}^{P}$ is world-selective, consider any $v \in W_{2}^{\omega_{1}}$. By conditions (ii) and (iii) of the definition of projections, there is a $p \in P$ such that $\operatorname{im}(p)=v$. By the construction of $\mathfrak{S}^{P}$, it suffices to show that $\operatorname{dom}(p)_{\left\langle W_{2}^{\omega 2}, \omega 2\right\rangle}^{\langle\rangle} Z_{p}^{\langle\rangle} v_{\left\langle W_{2}^{\omega_{1}}, \omega_{1}\right\rangle}^{\langle\rangle}$, which is routine. As noted above, $\mathfrak{M}_{\emptyset}^{P}$ is a $\times$ model by construction.

To see that $\mathfrak{M}_{\emptyset}^{P}$ is expressibly $\times$ closed, note that since $\mathfrak{M}$ is $\times$ closed, $\mathfrak{M} \vDash$ $\times \operatorname{Comp}_{C}$, and so by Theorem 2.13 and Proposition $1.2, \mathfrak{M}_{\emptyset}^{P} \vDash \times \operatorname{Comp}_{C}$. That $\mathfrak{M}_{\emptyset}^{P}$ is expressively $\times$ closed follows with Proposition 2.2 . 
It remains to show that $\mathfrak{M}_{\emptyset}^{P}$ is not $\times$ closed. Let $D$ and $D^{P}$ be the domain assignments of $\mathfrak{M}$ and $\mathfrak{M}_{\emptyset}^{P}$, respectively. Assume for contradiction that $\mathfrak{M}_{\emptyset}^{P}$ is

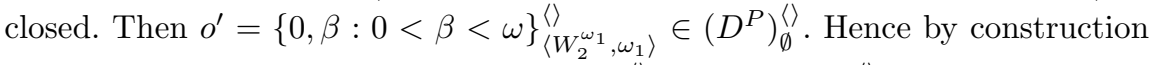
of $D^{P}$, there must be a $p \in P$ and $o \in D_{\emptyset}^{\langle\rangle}$such that $o Z_{p}^{\langle\rangle} o^{\prime}$. With condition (iii) of the definition of projections and Lemma 2.10, it follows that $o Z_{\emptyset}^{\langle\rangle} o^{\prime}$. But this conflicts with condition (2) of the definition of $Z$. $\downarrow$.

In this proof, two models verifying $\times \operatorname{Comp}_{C}$ are used, only one of which is $\times$ closed. It follows that $\times$ Comp $_{C}$ does not define $C \times$. Moreover, the two models satisfy the same sentences, so no class of sentences defines $C \times$, and since both models are world-selective, they lead to the following corollary:

Corollary 2.15. $C \times$ is undefinable relative to the class of world-selective models. I.e., there is no class of sentences $\Gamma$ such that a world-selective model $\mathfrak{M}$ is in $C \times$ if and only if $\mathfrak{M} \vDash \Gamma$.

Above, it was suggested that the results obtained in this section show that expressibility in principle cannot be cashed out in terms of expressibility in the infinitary higher-order modal language used here. One may wonder whether one could instead claim that in the relevant models, the relevant propositions are simply not expressible in principle, and so that it is the automorphism-theoretic condition which fails to capture expressibility in principle, rather than the condition of being expressible in infinitary higher-order modal logic. However, there is an intuitive sense in which the relevant propositions are expressible in principle: In the above model, it is plausible to think that the relevant uncountable proposition is conveyed (although maybe not literally expressed) by the sentence "There is something which could have co-existed, pairwise, with uncountably many possible individuals". What can be conveyed with this kind of loose talk seems to be expressible in principle as well. This observation also illustrates how the expressive limitations of this section are connected to the expressive limitations in the next section, where it will be shown that there is no way of paraphrasing claims which state that there are uncountably many possible individuals satisfying a given condition.

\section{Expressing Modalized Cardinality Claims}

Consider now what can be expressed given various theories of higher-order contingency. As argued in Fritz and Goodman (forthcoming), the relevant distinctions which are in need of being expressed relative to a certain theory of higherorder contingentism can be identified, at least for present purposes, with classes of models. Given a certain theory of higher-order contingency formalized using a class of models $X$, a claim, understood as the subclass $Y$ of $X$ in which it is true, is then expressible in a given language just in case there is a sentence in this language which is true in a model in $X$ if and only if it is in $Y$.

\subsection{Expressivity via Haecceities}

Recall the definition of existential and universal outer quantifiers in Part 1, section 5.3. Analogous to these defined outer quantifiers one can consider primitive generalized quantifiers which operate on the outer domain of models (see 
Fritz (2013) for this and other features of generalized quantifiers appealed to below). It will be shown how, assuming higher-order necessitism, such generalized quantifiers can always be eliminated in favour of the corresponding (inner) generalized quantifiers over properties, restricted to certain haecceities. Since the main focus of this paper is on the expressive limitations of higher-order contingentism, these constructions witnessing the expressive power of higher-order necessitism will only be sketched.

Higher-order necessitism will here be understood as formalized using the class of models in which necessarily, all relations exist which are compatible with the being constraint or its positive weakening; call these full models. Let $\mathcal{Q}$ be a generalized quantifier which binds a sequence of sequences of variables $\overline{\bar{x}}$ and operates on a sequence of formulas $\bar{\varphi}$ to yield a sentence of the form $\mathcal{Q} \overline{\bar{x}} \bar{\varphi}$. Assume first that the relativization of $\mathcal{Q}$, written $Q^{\text {rel }}$, is definable in infinitary higher-order non-modal logic, in the sense that there is a formula $\psi$ without occurrences of $\square$ or non-logical constants and with free variables $X, Y_{1}, \ldots, Y_{n}$ such that $\forall X Y_{1} \ldots Y_{n}\left(\psi \leftrightarrow Q^{\text {rel }} x \overline{\bar{y}}\left(X x, Y_{1} \bar{y}_{1}, \ldots, Y_{n} \bar{y}_{n}\right)\right)$ is true in all full models. In this case, the outer quantifier corresponding to $\mathcal{Q}$ can be expressed by turning $\varphi$ into a condition on haecceities instead of individuals.

To make this strategy precise, define first a mapping $\cdot{ }^{+}$on types, mapping $e$ to $\langle e\rangle$ and every complex type $\left\langle t_{1}, \ldots, t_{n}\right\rangle$ to $\left\langle t_{1}^{+}, \ldots, t_{n}^{+}\right\rangle$. Extend this to a mapping on non-modal formulas without non-logical constants which replaces every variable $X^{t}$ by $X^{\left(t^{+}\right)}$and $=$by $\stackrel{\text { HI }}{\sim}$. Define further what it is to be a haecceity of a possible individual $(\mathrm{H})$, what it is for a property of haecceities to contain a haecceity for every possible individual $(\mathrm{CH})$, and what it is for a relation among haecceities to relate exactly those haecceities of a given choice of representations which single out individuals which satisfy a given open formula (HMAP):

$$
\begin{aligned}
& \mathrm{H}\left(X^{\langle e\rangle}, x^{e}\right):=\square \forall y^{e} \square\left(X y \leftrightarrow\left(x=y \wedge \exists z^{e}(z=x)\right)\right) \\
& \mathrm{CH}\left(X^{\langle\langle e\rangle\rangle}\right):=\forall Y^{\langle e\rangle}\left(X Y \rightarrow \Sigma x^{e} \mathrm{H}(Y, x)\right) \wedge \Pi x^{e} \exists Y^{\langle e\rangle}(X Y \wedge \mathrm{H}(Y, x)) \\
& \operatorname{HMAP}\left(X^{\langle\langle e\rangle\rangle}, Y^{\langle e\rangle^{n}}, \varphi, \bar{x}^{e}\right):=\forall \bar{Z}^{\langle e\rangle} \Pi \bar{x}^{e}\left(\bigwedge_{i<n}\left(X Z_{i} \wedge \mathrm{H}\left(Z_{i}, x_{i}\right)\right) \rightarrow(Y \bar{Z} \leftrightarrow \varphi)\right)
\end{aligned}
$$

Then the outer quantifier corresponding to $\mathcal{Q}$ can be expressed as follows:

$$
Q^{*} \overline{\bar{x}}^{e} \bar{\varphi}:=\forall X^{\langle\langle e\rangle\rangle} Y_{1}^{\langle e\rangle^{k_{1}}} \ldots Y_{n}^{\langle e\rangle^{k_{n}}}\left(\left(\mathrm{CH}(X) \wedge \bigwedge_{i<n} \operatorname{HMAP}\left(X, Y_{i}, \varphi_{i}, \bar{x}_{i}\right)\right) \rightarrow \psi^{+}\right)
$$

What about generalized quantifiers which cannot be defined as assumed for $\mathcal{Q}$ ? Given the construction just sketched, one might assume that such quantifiers can at least be dealt with if the corresponding restricted quantifier among properties of individuals is added as a primitive logical constant $\left(Q^{\text {rel }}\right)^{\langle e\rangle}$. Indeed, this is so, but there is a subtlety to be noted. Since quantifiers in the language used here are implicitly restricted to hereditarily intensional entities, $\mathrm{CH}$ does not single out properties of haecceities which apply to a single haecceity for each possible individual, but all such haecceities. Above, this was no problem, since all higher-order quantifiers in the defining formula $\psi$ were restricted to hereditarily intensional relations. But the primitive generalized quantifier among properties of individuals cannot be assumed to only be sensitive to hereditarily intensional distinctions. Thus, the constraint expressed by $\mathrm{CH}$ has to be augmented by the condition that for each possible individual, the relevant choice 
of haecceities applies to only one of its haecceities. This requires predicating identity of haecceities, which is not possible in the restricted language. To do so, the unrestricted language of Part 1, section 3.4 is needed, where $X^{\langle e\rangle}=Y^{\langle e\rangle}$ can be read as abbreviating $\forall^{\mathcal{U}} Z^{\langle\langle e\rangle\rangle}(Z X \leftrightarrow Z Y)$ :

$U\left(X^{\langle\langle e\rangle\rangle}\right):=\forall Y^{\langle e\rangle} Z^{\langle e\rangle}\left(\left(X Y \wedge X Z \wedge \Sigma x^{e}(\mathrm{H}(Y, x) \wedge \mathrm{H}(Z, x)) \rightarrow Y=Z\right)\right.$

Similarly, $X$ must now be bound by a universal quantifier which is not restricted to hereditarily intensional relations. Otherwise the paraphrase strategy can be carried out as expected: $Q^{\dagger} \overline{\bar{x}}^{e} \bar{\varphi}:=\forall^{\mathcal{U}} X^{\langle\langle e\rangle\rangle} Y_{1}^{\langle e\rangle^{k_{1}}} \ldots Y_{n}^{\langle e\rangle^{k_{n}}}\left(\left(\mathrm{CH}(X) \wedge U(X) \wedge \bigwedge_{i<n} \operatorname{HMAP}\left(X, Y_{i}, \varphi_{i}, \bar{x}_{i}\right)\right) \rightarrow\right.$
$\left.\left(Q^{\mathrm{rel}}\right)^{\langle e\rangle} Z^{\langle e\rangle} \overline{\bar{Z}}^{\langle e\rangle}\left(X Z, Y_{1} \bar{Z}_{1}, \ldots, Y_{n} \bar{Z}_{n}\right)\right)$

\subsection{FFISs and Bi-Projections}

To show that analogous paraphrase strategies are not available given higherorder contingentism, as formalized using the classes of models developed in Part 1, the technique of projective generation is refined and used to construct back and forth systems between closed models. First, even stronger constraints will be imposed on FIS, forcing them to be fully symmetric in the sense of admitting each permutation of individuals as an automorphism:

Definition 3.1. An FIS $\mathfrak{S}=\langle W, I, D\rangle$ is fully symmetric if $\operatorname{aut}^{i}(\mathfrak{S})=S_{I}$. FFIS will be used to abbreviate finitary fully symmetric individual structure.

Note that every fully symmetric FIS is trivially homogeneous. One important feature of FFISs is that for purposes of generation, attention can be restricted to permutations with finite support, as will now be shown. (Recall that the support of a permutation is the set of elements it does not map to itself.)

Definition 3.2. Let $\mathfrak{S}=\langle W, I, D\rangle$ be an FIS and $w \in W$. Define:

- $\operatorname{aut}_{\omega}^{i}(\mathfrak{S})=\left\{f \in \operatorname{aut}^{i}(\mathfrak{S}): \operatorname{supp}(f)\right.$ is finite $\}$

- $\operatorname{fix}_{\omega}^{i}(\mathfrak{S}, w)=\left\{f \in \operatorname{fix}^{i}(\mathfrak{S}, w): \operatorname{supp}(f)\right.$ is finite $\}$

Lemma 3.3. Let $\mathfrak{S}$ be an FFIS and $\otimes \mathfrak{S}=\langle W, I, D\rangle$. Then for all types $t \neq e$, $w \in W$ and $o \in \iota_{\langle W, I\rangle}^{t}, o \in D_{w}^{t}$ iff $D \otimes o$ and $\hat{f} . o=o$ for all $f \in \operatorname{fix}_{\omega}^{i}(\mathfrak{S}, w)$.

Proof. The left-to-right direction is immediate. For the right-to-left direction, assume $o \notin D_{w}^{t}$ and $D \otimes o$. Then there is an $f \in \operatorname{fix}^{i}(\mathfrak{S}, w)$ and $\hat{f} . o \neq o$. So there are $v \in W, \bar{v} \in W^{n}$ and $\bar{o} \in \Pi_{i \leq n} D_{v_{i}}^{t_{i}}$ such that not $\hat{f} . \bar{o} \in o(\hat{f} . v)$ iff $\bar{o} \in o(v)$. Let $X=\bigcup\left\{v, v_{0}, \ldots, v_{n-1}, w\right\}$. Since $\mathfrak{S}$ is a FFIS, there is a $g \in \operatorname{fix}_{\omega}^{i}(\mathfrak{S}, w)$ such that $f|X=g| X$ and $\operatorname{supp}(g)$ is finite. Then $\hat{g} \cdot v=\hat{f} \cdot v ; \operatorname{similarly} \hat{g} \cdot \bar{v}=\hat{f} \cdot \bar{v}$ from which it is straightforward to derive that $\hat{g} \cdot \bar{o}=\hat{f} . \bar{o}$. So not $\hat{g} \cdot \bar{o} \in o(\hat{g} \cdot v)$ iff $\bar{o} \in o(v)$, and thus $\hat{g} . o \neq o$.

To be able to use projections to construct back and forth systems between closed models, it will be shown that if a projection from one FFIS to another is such that its inverse (the set of inverses of its perspectives) is a projection as well, then the structure it projectively generates is the structure generated by the second FFIS: 
Definition 3.4. A projection $P$ from an FIS $\mathfrak{S}$ to an FIS $\mathfrak{S}^{\prime}$ is a bi-projection if $P^{-1}=\left\{p^{-1}: p \in P\right\}$ is a projection from $\mathfrak{S}^{\prime}$ to $\mathfrak{S}$.

Theorem 3.5. If $\mathfrak{S}$ and $\mathfrak{S}^{\prime}$ are FFISs and $P$ is a bi-projection from $\mathfrak{S}$ to $\mathfrak{S}^{\prime}$, then $\otimes \mathfrak{S}^{\prime}$ is the structure projectively generated by $P$.

As it is somewhat complex, the proof is given in Appendix B. The condition of being a FFIS used here is of course extremely strong. This is mainly since it is simple and the structures which will be used below satisfy it, but the results of this section could very likely be proven on weaker assumptions.

\subsection{Inexpressibility of Cardinality Claims}

To show that various claims concerning the cardinality of possible individuals are not expressible over closed and internally closed structures, for every infinite cardinality $\kappa$, a highly symmetric structure with $\kappa$ individuals in the outer domain will be defined. To do so, an FIS will be defined on a set of cardinality $\kappa$, taking its finite subsets as the worlds. Such structures are clearly FFIS, and the structures they generate turn out to be internally closed. Moreover, any two such FFIS can be related by a bi-projection, and the models based on the structures they generate can be connected by a back and forth system, which makes it possible to show that no distinctions among infinite cardinalities of possible individuals can be drawn on internally closed, and so in particular also on closed, models. For further philosophical discussion of these models, see Fritz and Goodman (forthcoming, section 3.4). To define these models, write $X \subseteq_{\omega} Y$ for $X$ being a finite subset of $Y$.

Definition 3.6. For any set $X$, let $W_{\omega}^{X}=\left\{Y: Y \subseteq_{\omega} X\right\}, \mathfrak{F}_{\omega}^{X}=\left\langle W_{\omega}^{X}, X\right\rangle$ and $\mathfrak{S}_{\omega}^{X}$ the unique FIS determined by $W_{\omega}^{X}$ and $X$. Let $\mathfrak{M}_{\omega}^{X}$ be the model for the empty signature based on $\otimes \mathfrak{S}_{\omega}^{X}$ with distinguished world $\emptyset$.

Lemma 3.7. For any infinite set $X, \otimes \mathfrak{S}_{\omega}^{X}$ is internally $\times$ closed.

Proof. Let $\mathfrak{S}_{\omega}^{X}=\left\langle W_{\omega}^{X}, X, B\right\rangle$ and $\otimes \mathfrak{S}_{\omega}^{X}=\left\langle W_{\omega}^{X}, X, D\right\rangle$. Consider any $w \in$ $W_{\omega}^{X}$, type $t \neq e$ and $o \in \iota_{\mathfrak{F}_{\omega}^{X}}^{t}$. By Part 1, Proposition 16, it suffices to show that $o \in D_{w}^{t}$ iff $D \otimes o$ and $\xi . o \stackrel{=}{=} o$ for all $\left.\xi \in \operatorname{fix}\left(\otimes \mathfrak{S}_{\omega}^{X}, w\right)\right|_{c} \otimes \mathfrak{S}_{\omega}^{X}$. Since $\otimes \mathfrak{S}_{\omega}^{X}$ is a $\times$ structure, if $o \in D_{w}^{t}$ then $D \otimes o$, so the left to right direction is immediate. So assume that $o \notin D_{w}^{t}$ and $D \otimes o$. By Lemma 3.3, there is an $f \in \operatorname{fix}_{\omega}^{i}\left(\mathfrak{S}_{\omega}^{X}, w\right)$ such that $\hat{f} . o \neq o$. It only remains to show that $\left.\hat{f} \in \operatorname{fix}\left(\otimes \mathfrak{S}_{\omega}^{X}, w\right)\right|_{c} \otimes \mathfrak{S}_{\omega}^{X}$.

Since $f \in \operatorname{fix}_{\omega}^{i}\left(\mathfrak{S}_{\omega}^{X}, w\right), \hat{f} \in \operatorname{fix}\left(\mathfrak{S}_{\omega}^{X}, w\right)$, and so by Part 1, Lemma 3 (ii), $\hat{f} \in \operatorname{fix}\left(\otimes \mathfrak{S}_{\omega}^{X}, w\right)$. Also, since $f \in \operatorname{fix}_{\omega}^{i}\left(\mathfrak{S}_{\omega}^{X}, w\right), \operatorname{supp}(f)$ is finite, and therefore $\operatorname{supp}(f) \in W_{\omega}^{X}$. Define $f_{c} \in \iota_{\mathfrak{F}_{\omega}^{X}}^{\langle e, e\rangle}$ and $\dot{f}_{c} \in \iota_{\mathfrak{F}_{\omega}^{X}}^{\langle\langle\rangle,\langle\rangle\rangle}$such that for all $v \in W_{\omega}^{X}$ :

$$
\begin{aligned}
& f_{c}(v)= \begin{cases}\{\langle x, f(x)\rangle: x \in v\} & \text { if } \operatorname{supp}(f) \subseteq v \\
\emptyset & \text { otherwise }\end{cases} \\
& \dot{f}_{c}(v)= \begin{cases}\left\{\left\langle u_{\mathfrak{F}_{\omega}^{X}}^{\langle\rangle}, \dot{f}(u)_{\mathfrak{F}_{\omega}^{X}}^{\langle\rangle}\right\rangle: u \subseteq v\right\} & \text { if } \operatorname{supp}(f) \subseteq v \\
\emptyset & \text { otherwise }\end{cases}
\end{aligned}
$$

Using the fact that $g . f=f$ for any $g \in \operatorname{fix}_{\omega}^{i}\left(\mathfrak{S}_{\omega}^{X}, \operatorname{supp}(f)\right)$, it is routine to show that $f_{c} \in D_{\operatorname{supp}(f)}^{\langle e, e\rangle}$ and $\dot{f}_{c} \in D_{\operatorname{supp}(f)}^{\langle\langle\rangle,\langle\rangle\rangle}$. 
Theorem 3.8. For any infinite sets $X$ and $Y, \mathfrak{M}_{\omega}^{X} \cong \omega \mathfrak{M}_{\omega}^{Y}$.

Proof. Let $P$ be the set of partial injections from $X$ to $Y$ with finite domains. It is straightforward to see that $P$ is a bi-projection from $\mathfrak{S}_{\omega}^{X}$ to $\mathfrak{S}_{\omega}^{Y}$. So by Theorem 2.13, $J_{\emptyset}^{P}: \mathfrak{M}_{\omega}^{X} \cong \omega \mathfrak{M}_{\emptyset}^{P}$, where $J_{\emptyset}^{P}$ and $\mathfrak{M}_{\emptyset}^{P}$ are defined as above. By Theorem 3.5, $\mathfrak{M}_{\emptyset}^{P}$ is based on $\otimes \mathfrak{S}_{\omega}^{P}$, so $\mathfrak{M}_{\emptyset}^{P}=\mathfrak{M}_{\omega}^{Y}$, hence $J_{\emptyset}^{P}: \mathfrak{M}_{\omega}^{X} \cong \omega \mathfrak{M}_{\omega}^{Y}$.

Theorem 3.9. For any uncountable cardinality $\kappa$, there is no class of sentences $\Gamma$ such that for every internally $\times$ closed model $\mathfrak{M}=\langle W, I, D, V, w\rangle, \mathfrak{M} \vDash \Gamma$ if and only if $\left|\bigcup_{w \in W} D_{w}^{e}\right| \geq \kappa$.

Proof. Using Lemma 3.7, Theorem 3.8 and Proposition 1.2.

\section{Extending the Language}

In response to the expressive limitations seen above, one might suggest to enrich the language used there. One natural suggestion would be to lift the restriction of higher-order quantifiers to hereditarily intensional relations. This won't be considered in the following, simply because many aspects of the behaviour of higher-order modal logic are unclear without this restriction unless one makes the controversial assumption that the restriction is vacuous, in which case it is uninteresting to lift it. Similarly, adding hyperintensional operators as logical constants won't be considered. See Fritz and Goodman (forthcoming) for arguments that such resources are unpromising to deal with the problem of expressing modalized cardinality claims. Instead, this section considers two kinds of infinitary resources.

\subsection{Non-well-founded Languages}

Although the language $\mathcal{L}$ used here allows conjunctions of infinite sets of formulas and quantifiers binding infinite sets of variables, it is defined in the usual recursive manner. Consequently, although a node in a syntax tree of one of its formulas may have infinitely many immediate successors, this tree may not contain a path, following the successor relation, of infinite length. That is, such a tree may be infinitely branching, but it may not have infinite branches. This is equivalent to the condition that the subformula relation among formulas is well-founded, and in this sense, $\mathcal{L}$ may be called a well-founded language.

Since in such languages, formulas cannot contain infinite branches, every subformula is in the scope of only a finite number of modal operators. Therefore, in evaluating a sentence, a subformula will only be evaluated relative to an assignment which maps its free variables to parameters from a finite number of worlds. In this sense, it is impossible to say anything in these languages which requires comparing parameters from an infinite number of worlds; it is exactly this feature which both of the limitative results proven here exploit. In fact, it seems likely that an abstract characterization of the class of well-founded languages in which $\square$ is the only non-extensional operator can be given, and that both of the limitative theorems can be extended to any such language.

It is therefore natural to consider non-well-founded languages. As noted above, this is exactly what Fine does in appealing to languages in which infinite embeddings of operators are allowed. To evaluate the use of such languages, two 
questions have to be considered: first, whether they are in good standing, and second, whether they overcome the expressive limitations discussed here.

While Fine does not provide a formal syntax or a model-theoretic semantics for such a language, this is done in Leuenberger (2006) and Fritz and Goodman (forthcoming), building on Hintikka and Rantala (1976). Formally, these languages are therefore in good standing. But they may still be philosophically problematic, and arguments to this effect are given in Williamson (2013, section 7$)$.

Consider the question whether non-well-founded languages overcome the expressive limitations discussed here. Concerning the first issue of expressing the higher-order closure and qualitative generation view, it follows from results in Fine (1977b) that allowing infinite embeddings of operators suffices to establish the equivalence of the semantic and the linguistic existence criteria in Fine's formal setting. Given the details of his proof, it is not to be expected that the formal differences between this and the present setting will affect the result, and it is therefore to be expected that the analog of Theorem 2.14 or a similar result for generation do not hold for an extension of the language by infinitary embeddings. However, such a positive result would crucially rely on the fact that since models are based on sets, there are guaranteed to be formulas containing as many variables as individuals, which is arguably an artifact of the model theory; see the discussion in Fritz and Goodman (forthcoming, section 2.3). It is therefore doubtful whether the non-well-founded languages considered here address the first expressive limitation in a satisfactory way.

Consider now the case of paraphrasing claims formulated using modalized generalized quantifiers. As shown in Fritz and Goodman (forthcoming), infinitary embeddings suffice to paraphrase any claim formulated using modalized cardinality quantifiers (i.e., "there are $\kappa$ many possible $\varphi \mathrm{s}$ ", for any cardinality $\kappa)$, using only first-order quantifiers. But it is also shown there that in a firstorder language with such infinitary resources, claims of the form "most possible $\varphi$ s are possible $\psi \mathrm{s}$ " cannot be paraphrased. Can this be done if such a language is extended by higher-order quantifiers and interpreted over (internally) closed structures?

I conjecture that this is not the case: Even though for every cardinality $\kappa$, there are sentences in such a language which "collect and compare" possible individuals from $\kappa$ worlds, every particular sentence of such a language is itself a member of the set-theoretic hierarchy, and so can only "collect and compare" possible individuals from collections of worlds up to some particular cardinality. For a generalized quantifier like "most", no particular cardinality suffices, and there are always countermodels to a proposed paraphrase using sufficiently large models. While I won't attempt to do so here, I conjecture that this rough idea can be turned into a rigorous proof by combining the model-construction idea of section 3.3 with the syntactic approach of Fritz and Goodman (forthcoming, Appendix A.4). If this is right, then even on set-sized models, which introduce the arguably unrealistic artifact that formulas can contain as many variables as there are individuals, infinitary embeddings don't suffice to paraphrase "most possible $\varphi$ s are possible $\psi \mathrm{s} "$. 


\subsection{Transfinite Types}

As noted in Fine (1977b, p. 144), there are two dimensions along which the finitary type hierarchy explored here can be extended to admit transfinite types. On the one hand, one might extend the recursive definition of types by allowing relational types of arbitrary arity. On the other hand, one might admit cumulative types by adding in the recursive definition of types that each set of types $T^{\prime}$ is a type as well, understanding an entity to be of type $T^{\prime}$ just in case it is of a type which is a member of $T^{\prime}$. Conceptually, the former extension is much more natural, and the following therefore concentrates on this extension. It is important to note that extending the type hierarchy not only extends the formal language, but also the semantic structures on which they are interpreted.

Working in a positive setting, consider the following further extension of the language: For every sequence of types $\bar{t}$, add a logical constant $\approx$ of type $\langle\bar{t}, \bar{t}\rangle$, which expresses hereditary intensional equivalence, and a $\lambda$ operator binding a sequence of variables of types $\bar{t}$, where $\lambda \bar{v} \varphi$ is an expression of type $\bar{t}$ interpreted as the intension expressed by $\varphi$, abstracted over $\bar{v}$.

With these additional resources, Fine's infinitary outer quantifier is definable: letting $w^{\langle\rangle}$be the true world proposition (see Part 1, section 5.3), the universal outer quantification $\Pi \bar{v} \varphi$ is true if and only if $\operatorname{AT}(w, \varphi)$ and $T$, abstracted over $\bar{v}$, are hereditarily intensionally equivalent:

$$
\Pi \bar{v} \varphi:=\exists w^{\langle\rangle}(\operatorname{WORLD}(w) \wedge w \wedge(\lambda \bar{v} \operatorname{AT}(w, \varphi) \approx \lambda \bar{v} \top))
$$

This makes it possible to express infinitary outer quantification without appealing to the potentially problematic resources of non-well-founded languages discussed above. But as noted there, it is not clear that this will overcome either of the expressive limitations discussed here, likely leaving the higher-order contingentist unable to express their own view by cashing out talk of expressibility in principle by appealing to a particular infinitary language, as well as unable to express claims about most possible individuals.

\section{A Appendix on Projective Generation}

In this appendix, Lemma 2.10 is proven. To do so, two subsidiary lemmas are established.

Lemma A.1. Let $\mathfrak{S}=\langle W, I, D\rangle$ be an homogeneous FIS, $\mathfrak{S}^{\prime}=\left\langle W^{\prime}, I^{\prime}, D^{\prime}\right\rangle$ an FIS and $P$ a projection from $\mathfrak{S}$ to $\mathfrak{S}^{\prime}$. For all $p, q \in P$, there are $p^{\prime} \in P$ and $f \in \operatorname{aut}^{i}(\mathfrak{S})_{(\operatorname{dom}(p \cap q))}$ such that $p \subseteq p^{\prime}$ and $q \subseteq p^{\prime} f$.

Proof. By condition (ii) of projections, there is a $p^{\prime} \in P$ such that $p \subseteq p^{\prime}$ and $\operatorname{im}(q) \subseteq \operatorname{im}\left(p^{\prime}\right)$. Let $f$ be the partial function from $I$ to $I$ mapping every $x \in$ $\operatorname{dom}(q)$ to $p^{\prime-1} q(x)$. It is routine to show that $f$ is a finite partial permutation of $I$ which respects worlds. Since $\mathfrak{S}$ is homogeneous, there is an $f^{\prime} \in \operatorname{aut}^{i}(\mathfrak{S})$ such that $f \subseteq f^{\prime}$. It is routine to show that $f^{\prime} \in \operatorname{aut}^{i}(\mathfrak{S})_{(\operatorname{dom}(p \cap q))}$ and $q \subseteq p^{\prime} f^{\prime}$.

Lemma A.2. For any type $t, o \in D_{W}^{t}, o^{\prime} \in \iota_{\mathfrak{F}^{\prime}}^{t}, p \in P$ and $f \in \operatorname{aut}^{i}(\mathfrak{S})$, if $o Z_{p}^{t} o^{\prime}$ then $\hat{f} . o Z_{p f^{-1}}^{t} o^{\prime}$.

Proof. By induction on types. For $t=e$, note that if $o Z_{p}^{e} o^{\prime}$, then $p(o)=o^{\prime}$, so $p f^{-1} f(o)=o$, whence $\hat{f} . o Z_{p f^{-1}}^{e} o^{\prime}$. Let $t=\bar{t}$. To show that $\hat{f} . o Z_{p f^{-1}}^{t} o^{\prime}$, note 
that condition (1) follows from $o Z_{p}^{t} o^{\prime}$. For condition (2), consider any $q \in P$ such that $p f^{-1} \subseteq q, v \in \operatorname{dom}(\dot{q}), \bar{v} \in \operatorname{dom}(\dot{q})^{n}, \bar{o} \in \Pi_{i \leq n} D_{v_{i}}^{t_{i}}$, and $\bar{o}^{\prime} \in \Pi_{i \leq n} \iota_{\left\langle W^{\prime}, I^{\prime}\right\rangle}^{t_{i}}$ such that $\bar{o} Z_{q} \bar{o}^{\prime}$. It will be shown that $\bar{o} \in \hat{f} . o(v)$ iff $\bar{o}^{\prime} \in o^{\prime}(\dot{q}(v))$. By induction hypothesis, it follows from $\bar{o} Z_{q} \bar{o}^{\prime}$ that $\hat{f}^{-1} . \bar{o} Z_{q f} \bar{o}^{\prime}$. Note also that $p \subseteq q f$ (for any $\left.o \in \operatorname{dom}(p), p(o)=p f^{-1} f(o)=q f(o)\right)$. Thus it follows from $o Z_{p}^{t} o^{\prime}$ that $\hat{f}^{-1} . \bar{o} \in o\left(\hat{f}^{-1} . v\right)$ iff $\bar{o}^{\prime} \in o^{\prime}(\dot{q}(v))$. Since $\bar{o} \in \hat{f} . o(v)$ iff $\hat{f}^{-1} . \bar{o} \in o\left(\hat{f}^{-1} \cdot v\right)$, the desired equivalence follows.

Lemma 2.10 can now be proven, which claims that for all types $t$ and $p \in P$ :

(i) $Z_{p}^{t}$ is a bijection from $D_{\operatorname{dom}(\dot{p})}^{t}$ to $\left(D^{P}\right)_{\operatorname{im}(\dot{p})}^{t}$.

(ii) For all $q \in P, w \in W$ such that $w \subseteq \operatorname{dom}(p \cap q)$ and $o \in D_{w}^{t}, Z_{p}^{t}(o)=Z_{q}^{t}(o)$.

Proof of Lemma 2.10. By induction on types. The case of $t=e$ is trivial for both (i) and (ii). So consider any type $t=\bar{t}$ and $p \in P$. (i) will be established first:

Claim 1: $Z_{p}^{t}$ is functional. Proof. Consider any $o \in D_{\operatorname{dom}(\dot{p})}^{t}$ and $o^{\prime}, o^{\prime \prime} \in$ $\left(D^{P}\right)_{\operatorname{im}(\dot{p})}^{t}$ such that $o Z_{p}^{t} o^{\prime}$ and $o Z_{p}^{t} o^{\prime \prime}$. Let $v \in W^{\prime}$; it will be shown that $o^{\prime}(v) \subseteq$ $o^{\prime \prime}(v)$ (the other direction follows by symmetry). So consider any $\bar{o}^{\prime} \in o^{\prime}(v)$. Since $D^{P} \otimes o^{\prime}$, there are $\bar{v} \in W^{\prime n}$ such that $\bar{o}^{\prime} \in \Pi_{i \leq n}\left(D^{P}\right)_{v_{i}}^{t_{i}}$. By condition (ii) of the definition of projections, there is a $q \in P$ such that $p \subseteq q$ and $v, v_{1}, \ldots, v_{n} \in \operatorname{im}(\dot{q})$. By induction hypothesis (i), there are $\bar{o} \in \Pi_{i \leq n} D_{\operatorname{dom}(\dot{q})}^{t_{i}}$ such that $\bar{o} Z_{q} \bar{o}^{\prime}$. So it follows from $o Z_{p}^{t} o^{\prime}$ that $\bar{o} \in o\left(\dot{q}^{-1}(v)\right)$, and therefore with $o Z_{p}^{t} o^{\prime \prime}$ that $\bar{o}^{\prime} \in o^{\prime \prime}(v) \cdot \checkmark$

Claim 2: $Z_{p}^{t}$ is total. Proof. Consider any $o \in D_{\operatorname{dom}(\dot{p})}^{t}$. Define $o^{\prime} \in \iota_{\mathfrak{F}^{\prime}}^{t}$ such that for all $v \in W^{\prime}, o^{\prime}(v)$ is the set of $\bar{o}^{\prime} \in \Pi_{i \leq n}\left(D^{P}\right)_{W^{\prime}}^{t_{i}}($ if $\times=+) /$ $\bar{o}^{\prime} \in \Pi_{i \leq n}\left(D^{P}\right)_{v}^{t_{i}}$ (if $\left.\times=-\right)$ such that there is a $q \in P$ such that $p \subseteq q$ and $v \subseteq \operatorname{im}(q)$, and $n$-tuple $\bar{o}$ such that $\bar{o} Z_{q} \bar{o}^{\prime}$ and $\bar{o} \in o\left(\dot{q}^{-1}\left(v^{\prime}\right)\right)$. It will be shown that $o Z_{p}^{t} o^{\prime}$. $D^{P} \otimes o^{\prime}$ is immediate by construction. So consider any $q \in P$ such that $p \subseteq q, w \in \operatorname{dom}(\dot{q})$ and $n$-tuples $\bar{o}, \bar{o}^{\prime}$ such that $\bar{o} Z_{q} \bar{o}^{\prime}$. It will be proven that $\bar{o} \in o(w)$ iff $\bar{o}^{\prime} \in o^{\prime}(\dot{q}(w))$. By construction of $o^{\prime}$, the latter follows from the former. So assume that $\bar{o}^{\prime} \in o^{\prime}(\dot{q}(w))$. Then by construction of $o^{\prime}$, there is an $r \in P$ such that $p \subseteq r$ and $\dot{q}(w) \subseteq \operatorname{im}(r)$, and $n$-tuple $\bar{o}^{*}$ such that $\bar{o}^{*} Z_{r} \bar{o}^{\prime}$ and $\bar{o}^{*} \in o\left(\dot{r}^{-1} \dot{q}(w)\right)$. By Lemma A.1, there are $q^{\prime}, r^{\prime} \in P$ such that $q \subseteq q^{\prime}, r \subseteq r^{\prime}$ and an $f \in \operatorname{aut}^{i}(\mathfrak{S})_{(\operatorname{dom}(p))}$ such that $q^{\prime} f=r^{\prime}$. By induction hypothesis (ii), $\bar{o} Z_{q^{\prime}} \bar{o}^{\prime}$ and $\bar{o}^{*} Z_{r^{\prime}} \bar{o}^{\prime}$. Thus $\bar{o}^{*} Z_{q^{\prime} f} \bar{o}^{\prime}$, and so with Lemma A.2, $\hat{f} . \bar{o}^{*} Z_{q^{\prime}} \bar{o}^{\prime}$. By induction hypothesis (i), $Z_{q^{\prime}}^{t_{i}}$ is a bijection, for each $i \leq n$, so $\hat{f} . \bar{o}^{*}=\bar{o}$. Thus from the fact that $\bar{o}^{*} \in o\left(\dot{r}^{-1} \dot{q}(w)\right)$, we obtain $\bar{o} \in \hat{f} . o\left(\hat{f} . \dot{r}^{-1} \dot{q}(w)\right)$. Since $f \in \operatorname{aut}^{i}(\mathfrak{S})_{(\operatorname{dom}(p))}$ and $o \in D_{\operatorname{dom}(\dot{p})}^{t}, \hat{f} . o=o$, and so $\bar{o} \in o\left(\hat{f} \cdot \dot{r}^{-1} \dot{q}(w)\right)$, from which $\bar{o} \in o(w)$ follows with $q^{\prime} f=r^{\prime}$ as required. $\checkmark$

Claim 3: $Z_{p}^{t}$ is injective. Proof. Consider any $o, o^{*} \in D_{\operatorname{dom}(\dot{p})}^{t}$ and $o^{\prime} \in$ $\left(D^{P}\right)_{\operatorname{im}(\dot{p})}^{t}$ such that $o Z_{p}^{t} o^{\prime}$ and $o^{*} Z_{p}^{t} o^{\prime}$. Let $w \in W$; it will be shown that $o(w) \subseteq$ $o^{*}(w)$ (the other direction follows by symmetry). So consider any $\bar{o} \in o(w)$. By condition (i) of the definition of projections, there is a $q \in P$ such that $p \subseteq q$, $w \in \operatorname{dom}(\dot{q})$ and $\bar{o} \in \Pi_{i \leq n} D_{\operatorname{dom}(\dot{q})}^{t_{i}}$. So by induction hypothesis (i), there are $\bar{o}^{\prime} \in \Pi_{i \leq n}\left(D^{P}\right)_{\operatorname{im}(\dot{q})}^{t_{i}}$ such that $\bar{o}^{\prime} \in o^{\prime}(\dot{q}(w))$, and so $\bar{o} \in o^{*}(w) \cdot \checkmark$ 
Claim 4: $Z_{p}^{t}$ is surjective. Proof. Consider any $o^{\prime} \in\left(D^{P}\right)_{\operatorname{im}(\dot{p})}^{t}$. By construction of $D^{P}$, there is a $v \in \operatorname{im}(\dot{p})$ and $q \in P$ such that $v \subseteq \operatorname{im}(q)$ and $o^{\prime} \in Z_{q}^{t}\left[D_{\dot{q}^{-1}(v)}^{t}\right]$. Hence there is an $o \in D_{\dot{q}^{-1}(v)}^{t}$ such that $o Z_{q}^{t} o^{\prime}$. By Lemma A.1, there are $p^{\prime}, q^{\prime} \in P$ such that $p \subseteq p^{\prime}, q \subseteq q^{\prime}$, and an $f \in \operatorname{aut}^{i}(\mathfrak{S})$ such that $p^{\prime} f=q^{\prime}$. So by Lemma A.2, f..$o Z_{q^{\prime} f^{-1}}^{t} o^{\prime}$, hence $\hat{f} . o Z_{p^{\prime}}^{t} o^{\prime}$. Since $o \in D_{\dot{q}^{-1}(v)}^{t}$, $\hat{f} . o \in D_{\hat{f} . q^{-1}(v)}^{t}=D_{p^{-1}(v)}^{t}$. So $\hat{f} . o \in \operatorname{dom}\left(Z_{p}^{t}\right)$, and thus with induction hypothesis (ii), $\hat{f} . o Z_{p}^{t} o^{\prime}$. Hence $o^{\prime} \in \operatorname{im}\left(Z_{p}^{t}\right)$ as required.

(ii): Since (i) has been established, $Z_{p}^{t}$ must be a bijection from $D_{\operatorname{dom}(\dot{p})}^{t}$ to $\left(D^{P}\right)_{\operatorname{im}(\dot{p})}^{t}$, for all $o \in P$. Note first that it is routine to show that for all $p, q \in P$, if $p \subseteq q$ then $Z_{p}^{t} \subseteq Z_{q}^{t}$. Now consider any $p, q \in P, w \in W$ such that $w \subseteq \operatorname{dom}(p \cap q)$ and $o \in D_{w}^{t}$; it will be shown that $Z_{p}^{t}(o)=Z_{q}^{t}(o)$. By Lemma A.1, there are $p^{\prime}, q^{\prime} \in P$ and $f \in \operatorname{aut}^{i}(\mathfrak{S})_{(w)}$ such that $p \subseteq p^{\prime}, q \subseteq q^{\prime}$ and $p^{\prime} f=q^{\prime}$. Since $Z_{p} \subseteq Z_{p^{\prime}}, Z_{p}^{t}(o)=Z_{p^{\prime}}^{t}(o)$, which by Lemma A.2 is $Z_{p^{\prime} f}^{t}\left(\hat{f}^{-1} . o\right)$. Since $o \in D_{w}^{t}, \hat{f}^{-1} . o=o$, so $Z_{p}^{t}(o)=Z_{p^{\prime} f}^{t}(o)$, which is $Z_{q^{\prime}}^{t}(o)$. With the fact that $Z_{q}^{t} \subseteq Z_{q^{\prime}}^{t}$, it follows that $Z_{p}^{t}(o)=Z_{q}^{t}(o)$.

\section{B Appendix on FFISs and Bi-Projections}

This appendix proves Theorem 3.5. For the following, let $\mathfrak{S}=\langle W, I, B\rangle$ and $\mathfrak{S}^{\prime}=\left\langle W^{\prime}, I^{\prime}, B^{\prime}\right\rangle$ be FFIS, and $P$ a bi-projection from $\mathfrak{S}$ to $\mathfrak{S}^{\prime}$. Let $D$ and $D^{\prime}$ be the domain assignments of $\otimes \mathfrak{S}$ and $\otimes \mathfrak{S}^{\prime}, Z$ the extension of $P$ as defined above, and $D^{P^{-1}}$ and $D^{P}$ the domain assignments of the structures projectively generated by $P^{-1}$ and $P$, respectively.

Definition B.1. For any $p \in P$, define a relation $Z_{p}^{p} \subseteq \operatorname{aut}_{\omega}^{i}(\mathfrak{S}) \times \operatorname{aut}_{\omega}^{i}\left(\mathfrak{S}^{\prime}\right)$ such that for all $f \in \operatorname{aut}_{\omega}^{i}(\mathfrak{S})$ and $g \in \operatorname{aut}_{\omega}^{i}\left(\mathfrak{S}^{\prime}\right), f Z_{p}^{p} g$ iff

$$
\begin{aligned}
& \operatorname{supp}(f) \subseteq \operatorname{dom}(p), \\
& \operatorname{supp}(g) \subseteq \operatorname{im}(p), \text { and } \\
& p f(o)=g p(o) \text { for all } o \in \operatorname{supp}(f) .
\end{aligned}
$$

Lemma B.2. For any $p \in P, w \in \operatorname{dom}(\dot{p})$ and $f \in \mathrm{fix}_{\omega}^{i}\left(\mathfrak{S}^{\prime}, \dot{p}(w)\right)$ such that $\operatorname{supp}(f) \subseteq \operatorname{im}(p)$, there is a $g \in \operatorname{fix}_{\omega}^{i}(\mathfrak{S}, w)$ such that $g Z_{p}^{p} f$.

Proof. Define $g: I \rightarrow I$ such that for all $o \in I$,

$$
g(o)= \begin{cases}p^{-1} f p(o) & \text { if } o \in \operatorname{dom}(p) \\ o & \text { otherwise }\end{cases}
$$

It is routine to check that $g \in \operatorname{fix}_{\omega}^{i}(\mathfrak{S}, w)$ and $g Z_{p}^{p} f$.

Lemma B.3. Let $p, q \in P, f \in \operatorname{aut}_{\omega}^{i}(\mathfrak{S})$ and $g \in \operatorname{aut}_{\omega}^{i}\left(\mathfrak{S}^{\prime}\right)$. If $f Z_{p}^{p} g$ and $p \subseteq q$ then $f^{-1} Z_{q}^{p} g^{-1}$.

Proof. Routine.

Lemma B.4. For all types $t$ : 
(i) For all $p \in P, f \in \operatorname{aut}_{\omega}^{i}(\mathfrak{S}), g \in \operatorname{aut}_{\omega}^{i}\left(\mathfrak{S}^{\prime}\right)$ such that $f Z_{p}^{p} g$ and $\left\langle o, o^{\prime}\right\rangle \in Z_{p}^{t}$, $\hat{f} . o Z_{p}^{t} \hat{g} . o^{\prime}$.

(ii) For all $p \in P,\left(Z_{p}^{t}\right)^{-1}=Z_{p^{-1}}^{t}$.

(iii) For all $v \in W^{\prime}, D_{v}^{\prime t}=\left(D^{P}\right)_{v}^{t}$.

Proof. By induction on types. Let $t=e$. (i): Let $p \in P, f \in \operatorname{aut}_{\omega}^{i}(\mathfrak{S}), g \in$ aut $_{\omega}^{i}\left(\mathfrak{S}^{\prime}\right)$ such that $f Z_{p}^{p} g$ and $\left\langle o, o^{\prime}\right\rangle \in Z_{p}^{e}$. Then $o \in \operatorname{dom}(p)$, so $p(o)=o^{\prime}$. Since $f Z_{p}^{p} g, p f(o)=g p(o)$, so $p f(o)=g\left(o^{\prime}\right)$, i.e., $\hat{f} . o Z_{p}^{e} \hat{g} . o^{\prime}$. (ii) and (iii) are immediate. So let $t=\bar{t}$.

(i): Consider any $p \in P, f \in \operatorname{aut}_{\omega}^{i}(\mathfrak{S}), g \in \operatorname{aut}_{\omega}^{i}\left(\mathfrak{S}^{\prime}\right)$ such that $f Z_{p}^{p} g$ and $\left\langle o, o^{\prime}\right\rangle \in Z_{p}^{t}$. Then $\hat{f} . o \in D_{\hat{f} \text {.dom }(\dot{p})}^{t}$. Since $\operatorname{supp}(f) \subseteq \operatorname{dom}(p), \hat{f} . \operatorname{dom}(\dot{p})=$ $\operatorname{dom}(\dot{p})$, so $\hat{f} . o \in D_{\operatorname{dom}(\dot{p})}^{t}$. It will be shown that $\hat{f} . o Z_{p}^{t} \hat{g} . o^{\prime}$ by checking conditions (1) and (2) of the construction of $Z$.

(1): Consider any $v \in W^{\prime}$ and $\bar{o}^{\prime} \in \hat{g} \cdot o^{\prime}(v)$. Then $\hat{g}^{-1} \cdot \bar{o}^{\prime} \in o^{\prime}\left(\hat{g}^{-1} \cdot v\right)$. Since $o Z_{p}^{t} o^{\prime}, D^{P} \otimes o^{\prime}$, so there are $\bar{v} \in W^{\prime n}$ such that $\hat{g}^{-1} \cdot \bar{o}^{\prime} \in \Pi_{i \leq n}\left(D^{P}\right)_{v_{i}}^{t_{i}} \cdot g \in$ $\operatorname{aut}_{\omega}^{i}\left(\mathfrak{S}^{\prime}\right)$, so by Part 1, Lemma 3 (i), $\hat{g} \in \operatorname{aut}\left(\otimes \mathfrak{S}^{\prime}\right)$. Since by IH (iii), $\left(D^{P}\right)_{v_{i}}^{t_{i}}=$ $D_{v_{i}}^{\prime t_{i}}$ for all $i \leq n$, it follows that $\bar{o}^{\prime} \in \Pi_{i \leq n}\left(D^{P}\right)_{\hat{g} . v_{i}}^{t_{i}}$. If $\times=-$, it can be assumed that $v_{i}=v$ for all $i<n$, and therefore $\bar{o}^{\prime} \in \Pi_{i \leq n}\left(D^{P}\right)_{\hat{g} \cdot v}^{t_{i}}$. Thus $D^{P} \otimes \bar{o}^{\prime}$.

(2): Consider any $q \in P$ such that $p \subseteq q, w \in \operatorname{dom}(\dot{q})$ and $n$-tuples $\bar{o}, \bar{o}^{\prime}$ such that $\bar{o} Z_{q} \bar{o}^{\prime}$. It will be proven that $\bar{o} \in \hat{f} . o(w)$ iff $\bar{o}^{\prime} \in \hat{g} . o^{\prime}(\dot{q}(w))$. By Lemma B.3, $f^{-1} Z_{q}^{p} g^{-1}$, so by IH (i), $\hat{f}^{-1} \cdot \bar{o} Z_{q} \hat{g}^{-1} \cdot \bar{o}^{\prime}$. Since $\operatorname{supp}(f) \subseteq \operatorname{dom}(\dot{q}), \hat{f}^{-1} \cdot w \in$ $\operatorname{dom}(\dot{q})$, so $\hat{f}^{-1} \cdot \bar{o} \in o\left(\hat{f}^{-1} \cdot w\right)$ iff $\hat{g}^{-1} \cdot \bar{o}^{\prime} \in o^{\prime}\left(\dot{q}\left(\hat{f}^{-1} \cdot w\right)\right)$, and so $\bar{o} \in \hat{f} . o(w)$ iff $\bar{o}^{\prime} \in \hat{g} \cdot o^{\prime}\left(\hat{g} \cdot \dot{q}\left(\hat{f}^{-1} \cdot w\right)\right) \cdot q f=g q$, so $\hat{g} \cdot \dot{q}\left(\hat{f}^{-1} \cdot w\right)=\dot{q}(w)$, from which the desired claim follows.

(ii): Let $p \in P$. By symmetry, it suffices to show that if $\left\langle o, o^{\prime}\right\rangle \in Z_{p}^{t}$ then $\left\langle o^{\prime}, o\right\rangle \in Z_{p^{-1}}^{t}$. So assume $\left\langle o, o^{\prime}\right\rangle \in Z_{p}^{t}$. Then there is a $w \in \operatorname{dom}(\dot{p})$ such that $o \in D_{w}^{t}$ and $o^{\prime} \in\left(D^{P}\right)_{\dot{p}(w)}^{t}$. It will first be shown that $o^{\prime} \in D_{\dot{p}(w)}^{\prime t}$. By Lemma 3.3, it suffices to show that $D^{\prime} \otimes o^{\prime}$ and $\hat{f} . o^{\prime}=o^{\prime}$ for all $f \in \operatorname{fix}_{\omega}^{i}\left(\mathfrak{S}^{\prime}, \dot{p}(w)\right)$. By Lemma 2.10 (ii), $o^{\prime} \in\left(D^{P}\right)_{\mathrm{im}(\dot{p})}^{t}$, so with the fact that the structure projectively generated by $P$ is a $\times$ structure, it follows that $D^{P} \otimes o^{\prime}$. Therefore by IH (iii), $D^{\prime} \otimes o^{\prime}$. So consider any $f \in \operatorname{fix}_{\omega}^{i}\left(\mathfrak{S}^{\prime}, \dot{p}(w)\right)$. Since $\operatorname{supp}(f)$ is finite, there is a $q \in P$ such that $p \subseteq q$ and $\operatorname{supp}(f) \subseteq \operatorname{im}(q)$. So by Lemma B.2, there is a $g \in \operatorname{fix}_{\omega}^{i}(\mathfrak{S}, w)$ such that $g Z_{q}^{p} f$. By Lemma 2.10 (ii), $o Z_{q}^{t} o^{\prime}$, so with claim (i) of the present lemma, $\hat{g} . o Z_{q}^{t} \hat{f} . o^{\prime}$. As $o \in D_{w}^{t}, \hat{g} . o=o$, so $o Z_{q}^{t} \hat{f} . o^{\prime}$. Hence by the functionality of $Z_{q}^{t}$, established in Lemma 2.10 (i), $\hat{f} . o^{\prime}=o^{\prime}$.

With $o^{\prime} \in D_{\dot{p}(w)}^{\prime \prime}$ established, it can be proven that $o^{\prime} Z_{p^{-1}}^{t} o$ by checking conditions (1) and (2) of the definition of $Z$. Since $o \in D_{w}^{t}, D \otimes o$, so by IH, $D^{P^{-1}} \bowtie o$. For condition (2), consider any $q \in P^{-1}$ such that $p^{-1} \subseteq q, v \in \operatorname{dom}(\dot{q})$ and $n$-tuples $\bar{o}, \bar{o}^{\prime}$ such that $\bar{o} Z_{q} \bar{o}^{\prime}$. By IH, $\bar{o}^{\prime} Z_{q^{-1}} \bar{o}$; also $q^{-1} \in P, p \subseteq q^{-1}$ and $\dot{q}(v) \in \operatorname{dom}\left(\dot{q}^{-1}\right)$. So by $o Z_{p}^{t} o^{\prime}, \bar{o}^{\prime} \in o(\dot{q}(v))$ iff $\bar{o} \in o^{\prime}(v)$, as required for condition (2). So $o^{\prime} Z_{p^{-1}}^{t} o$.

(iii): Let $v \in W^{\prime}$. By condition (iii) of the definition of projections, there is a $p \in P$ such that $v \in \operatorname{im}(\dot{p})$. By Lemma $2.11, D_{v}^{\prime t}=\operatorname{dom}\left(Z_{p^{-1} \mid v}^{t}\right)=$ $\operatorname{dom}\left(Z_{\left(p \mid p^{-1}(v)\right)^{-1}}^{t}\right)$. By (ii), this is $\operatorname{im}\left(Z_{p \mid p^{-1}(v)}^{t}\right)$, which by Lemma 2.11 again 
is $\operatorname{im}\left(Z_{p}^{t} \mid D_{p^{-1}(v)}^{t}\right)$. By Lemma $2.10(\mathrm{i})$, this is $\left(D^{P}\right)_{v}^{t}$.

Proof of Theorem 3.5. Immediate by Lemma B.4.

\section{Acknowledgements}

In addition to those thanked in the acknowledgements of Part 1, I would like to thank a reviewer for comments on Part 3, and the editor, Frank Veltman, for all his help with the publication of the three parts.

\section{References}

Robert Merrihew Adams. Primitive thisness and primitive identity. The Journal of Philosophy, 76(1):5-26, 1979.

Patrick Blackburn, Maarten de Rijke, and Yde Venema. Modal Logic, volume 53 of Cambridge Tracts in Theoretical Computer Science. Cambridge: Cambridge University Press, 2001.

Kit Fine. Postscript to Worlds, Times and Selves (with A. N. Prior). London: Duckworth, 1977a.

Kit Fine. Properties, propositions and sets. Journal of Philosophical Logic, 6 (1):135-191, 1977b.

Kit Fine. The problem of possibilia. In Michael J. Loux and Dean W. Zimmerman, editors, The Oxford Handbook of Metaphysics, pages 161-179. Oxford: Oxford University Press, 2003.

Roland Fraïssé. Sur une extension de la polyrelation et des parentés tirant son origine $\mathrm{du}$ calcul logiques $\mathrm{du}$ k-ème échelon. In Le raisonnement en mathématiques et en sciences expérimentales, volume 70 of Colloques Internationaux du CNRS, pages 45-50. Paris: Editions du Centre National de la Recherche Scientifique, 1958.

Peter Fritz. Modal ontology and generalized quantifiers. Journal of Philosophical Logic, 42(4):643-678, 2013.

Peter Fritz. Higher-order contingentism, part 2: Patterns of indistinguishability. Journal of Philosophical Logic, forthcoming.

Peter Fritz and Jeremy Goodman. Higher-order contingentism, part 1: Closure and generation. Journal of Philosophical Logic, 45(6):645-695, 2016.

Peter Fritz and Jeremy Goodman. Counting incompossibles. Mind, forthcoming.

Jaakko Hintikka and Veikko Rantala. A new approach to infinitary languages. Annals of Mathematical Logic, 10(1):95-115, 1976.

Wilfrid Hodges. A Shorter Model Theory. Cambridge: Cambridge University Press, 1997. 
Stephan Leuenberger. A new problem of descriptive power. The Journal of Philosophy, 103(3):145-162, 2006.

David Lewis. On the Plurality of Worlds. Oxford: Basil Blackwell, 1986.

Robert Stalnaker. Mere Possibilities. Princeton: Princeton University Press, 2012.

Timothy Williamson. Modal Logic as Metaphysics. Oxford: Oxford University Press, 2013. 